\title{
Synthesis, Structure, and Biological Screening of Some Condensed Heterocyclic Compounds from 8-Chlorotetrazolo [5,1- f]-1,2,4-Triazine Precursor
}

\author{
Mamdouh A. M. Taha \\ Department of Chemistry, Faculty of Science, Fayoum University, Fayoum 63514, Egypt
}

\begin{abstract}
Chlorotetrazolo [5,1-f]-1,2,4-triazine (2) reacted with different compounds to yield a variety of novel condensed heterocyclic nitrogen compounds. The newly prepared structures were characterized by spectral data and screened for their antimicrobial activities against bacteria and fungi strains.
\end{abstract}

Keywords: 8-Chlorotetrazolo [5,1-f]-1,2,4-triazine, reactions, condensed heterocyclic compounds, antimicrobial assessment

\section{INTRODUCTION}

Many compounds consisting of the tetrazole nucleus have received ${ }^{1-10}$ much great attention because of their wide range of therapeutic and biological properties. ${ }^{11,12}$ They have emerged as antibacterial, ${ }^{2-8,13}$ antiproliferation, ${ }^{14}$ anticancer, ${ }^{14}$ and anticonvulsant ${ }^{15}$ agents. In this article, it is our intention to enlarge the area of the investigation towards tetrazolo-heterocycles using framework different reagants and expected interesting antimicrobial agents.

\section{RESULTS AND DISCUSSION}

The IR spectrum of tetrazolo[5,1-f]-1,2,4-triazin- $8(7 \mathrm{H})$ - one $^{4}(\mathbf{1})$ showed a characteristic absorption bands at 3260 and $1670 \mathrm{~cm}^{-1}$ corresponding to the $\mathrm{NH}$ and CONH groups and the ${ }^{1} \mathrm{H}$ NMR spectrum revealed exchangeable $\mathrm{NH}$ signal at $11.81 \mathrm{ppm}$. Compound 1 was treated with a mixture of phosphorous pentachloride and phosphorous oxychloride to yield the corresponding 8chlorotetrazolo[5,1-f]-1,2,4-triazine (2) and was confirmed on the basis of its elemental analysis and devoid any bands for $\mathrm{NH}$ and $\mathrm{CONH}$ groups in IR region. The structure of compound $\mathbf{2}$ is a promising for the synthesis of diverse condensed heterocyclic nitrogen compounds (Schemes $\mathbf{1}$ and $\mathbf{2}$ ).

Treatment of cyclic imidoyl chloride $\mathbf{2}$ with the sodium salt of various amino acids namely: glycine, D-alanine, and $\beta$-alanine under reflux conditions produced the corresponding tetrazolotriazinylamino acids $3 a, b$ and $\mathbf{4}$. The IR spectra of the latter compounds were confirmed on the basis absorption for $\mathrm{OH}, \mathrm{NH}$ and $\mathrm{CO}$ groups. The $\quad{ }^{1} \mathrm{H}$ NMR spectrum of compound $\mathbf{3} a$ displayed $\mathrm{CH}_{2}$ protons as a singlet signal at $4.56 \mathrm{ppm}$ and exchangeable $\mathrm{OH}$ and $\mathrm{NH}$ protons as singlet signals at 11.21 and 8.12 ppm, respectively. The ${ }^{1} \mathrm{H}$ NMR spectrum of compound $3 b$ displayed a $\mathrm{CH}$ proton as a quartet signal at $4.42 \mathrm{ppm}$ and $\mathrm{CH}_{3}$ protons as a doublet signal at $1.51 \mathrm{ppm}$. The ${ }^{1} \mathrm{H}$ NMR spectrum of compound 4 showed a triplet signal at $3.62 \mathrm{ppm}$ assigned for $\mathrm{CH}_{2} \mathrm{CO}$ and triplet signal at $3.22 \mathrm{ppm}$ assigned for $\mathrm{NCH}_{2}$ beside exchangeable $\mathrm{OH}$ and $\mathrm{NH}$ protons. The mass spectra of $\mathbf{3} a, b$ and $\mathbf{4}$ revealed the correct molecular ions which were supported by elemental analysis. The amino acid derivatives $\mathbf{3} a, b$ and $\mathbf{4}$ were easily cyclized ${ }^{16}$ via 1,3 tautomerism in heating acetic anhydride in the presence of anhydrous sodium acetate to give imidazotetrazolotriazine derivatives $\mathbf{5} a, b$ and tetrazolopyrimidinotriazine $\mathbf{6}$. The IR region of these compounds displayed the disappearance $\mathrm{OH}, \mathrm{NH}$ and $\mathrm{CO}$ absorptions and showed of the absorption bands for amide groups at 1670-1680 $\mathrm{cm}^{-1}$. The ${ }^{1} \mathrm{H}$ NMR spectra of compounds $5 a, b$ and $\mathbf{6}$ showed the absence of $\mathrm{OH}$ and $\mathrm{NH}$ signals characteristic of the parent amino acids $\mathbf{3} a, b$ and $\mathbf{4}$. These data together with the correct elemental analysis are compatible with $\mathbf{5} a, b$ and 6 structures.

The reaction of chloro compound $\mathbf{2}$ with variety of aromatic acid hydrazides namely: benzoic, $p$ toluic, $p$-chlorobenzoic, and $p$-nitrobenzoic afforded 3-aryl-1,2,4-triazolo[4,3- $d]$ tetrazolo [5,1-f]-1,2,4triazine derivatives $7 a-d$, respectively. Elemental and spectra data of the latter compounds are consistent with the structure assigned to their compounds ( $c f$. Experimental). More recently in the 
literature, ${ }^{4}$ the aforementioned triazolotetrazotriazine structures were constructed by the reaction of 8 hydrazinotetrazole $[5,1-f]-1,2,4$-triazine with triethyl orthoformate or glacial acetic acid.<smiles>O=c1[nH]cnn2nnnc12</smiles>

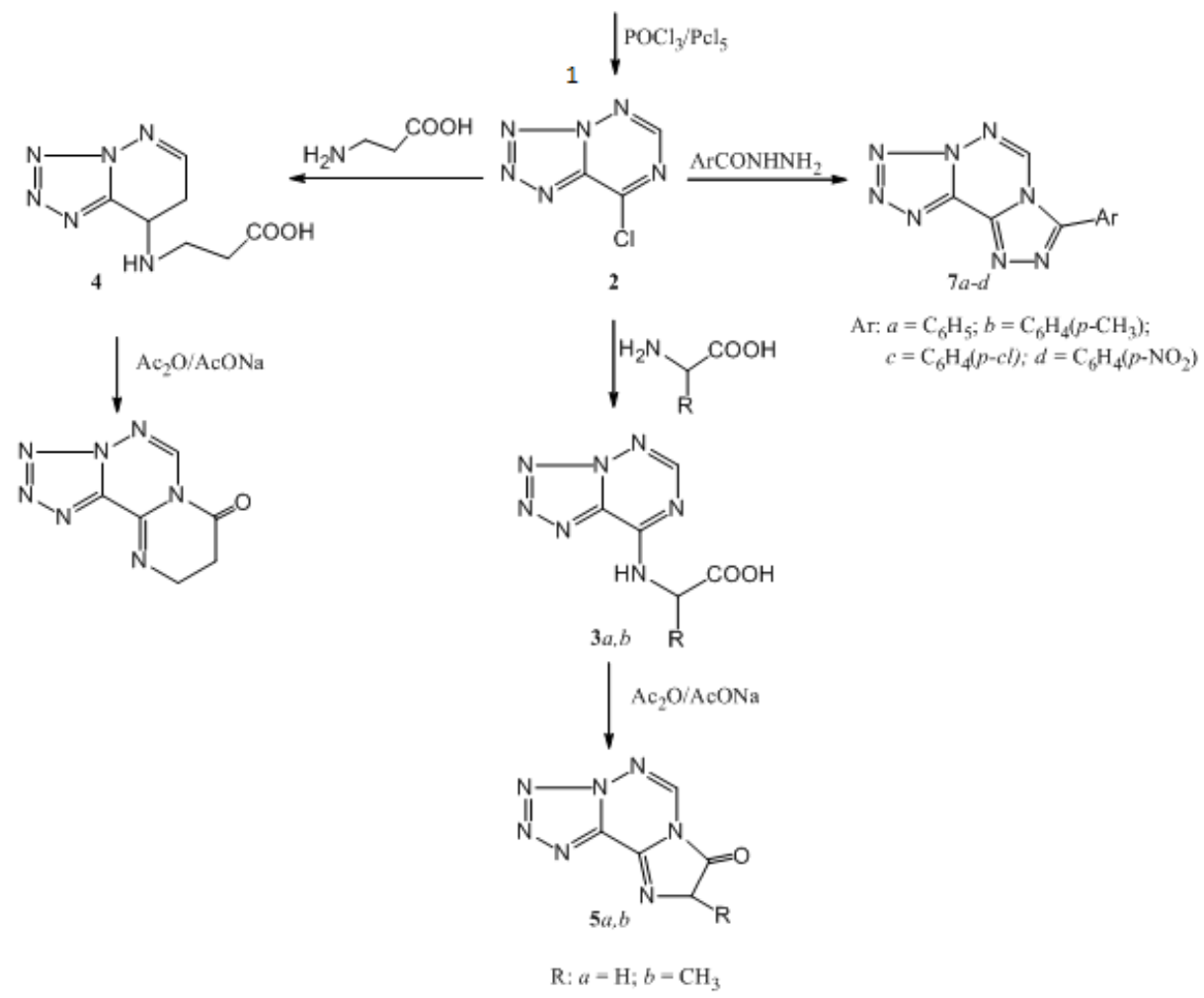

Scheme1

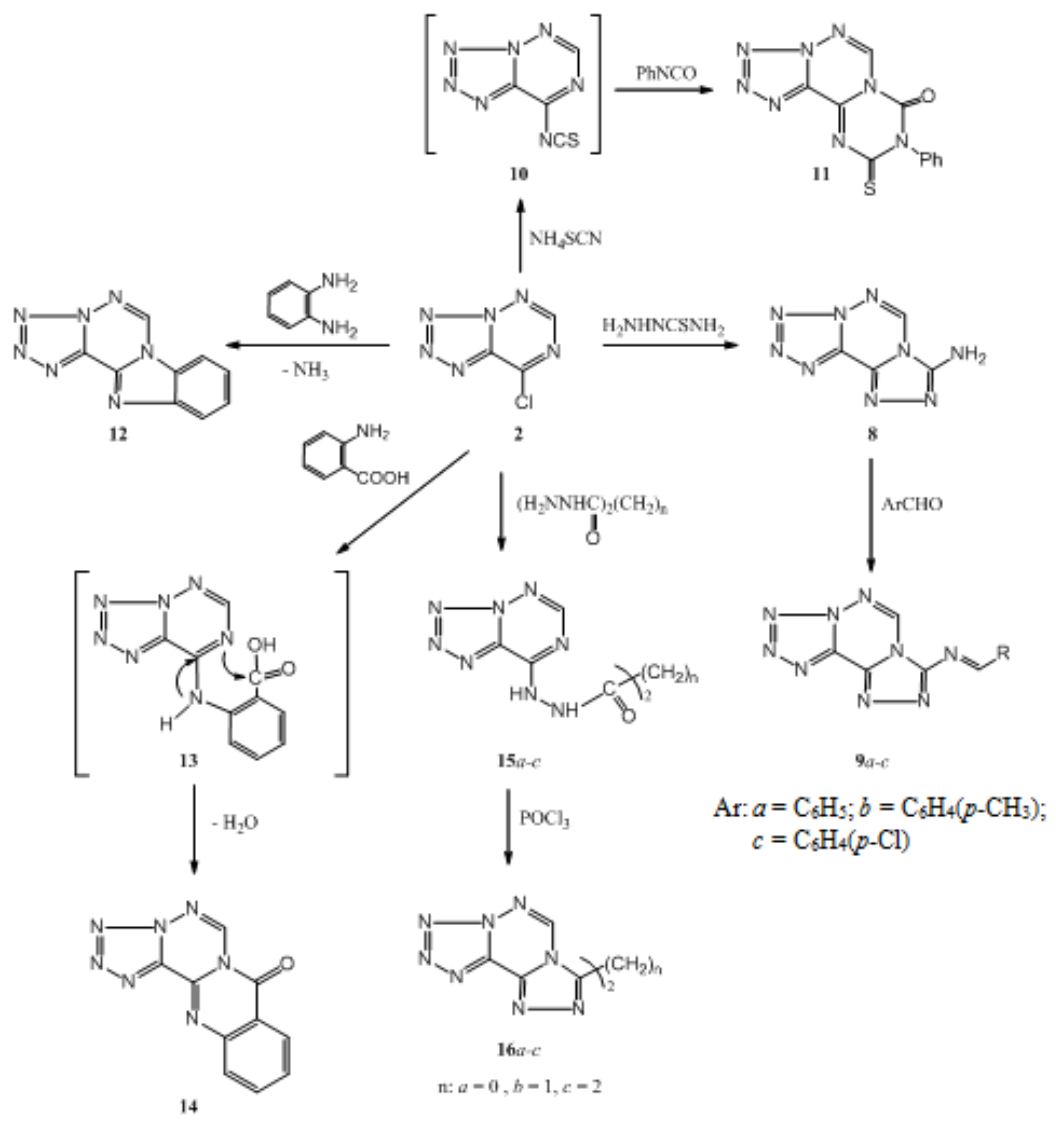

Scheme2 
Cyclization of cyclic imidoyl chloride 2 using thiosemicarbazide in ethanol yielded 3-amino-1,2,4triazolo derivative 8 . The ${ }^{1} \mathrm{H}$ NMR spectrum of compound $\mathbf{8}$ displayed two protons assigned exchangeable $\mathrm{NH}_{2}$ as a singlet signal at $6.30 \mathrm{ppm}$. Additionally, the condensation of $\mathbf{8}$ with different aromatic aldehydes namely: benzaldehyde, $p$-tolualdehyde, and $p$-chlorobenzaldehyde leading to the arylidenes $9 a-c$, respectively. The IR spectra of the latter compounds possessed a characteristic absorption bands at 1620 and $1628 \mathrm{~cm}^{-1}$ corresponding to the $\mathrm{C}=\mathrm{N}$ groups. The ${ }^{1} \mathrm{H}$ NMR spectra of compounds $9 a-c$ revealed the presence azomethine $(\mathrm{CH}=\mathrm{N})$ at $\delta 8.52$ and $9.02 \mathrm{ppm}$.

Furthermore, the reaction of chloro compound $\mathbf{2}$ with ammonium thiocyanate in ethanol produced the unisolable intermediate $\mathbf{1 0}$ that reacted in situ with phenyl isocyanate via $2+4$ cycloaddition reaction to build 3-phenyl-2-thioxo-2,3-dihydrotetrazolo[5 '1 $\left.1^{\prime}: 6,1\right]-1,2,4$-triazino[4,5- $\left.a\right]$-1,3,5-triazin-4one(11). The IR spectrum of 11 showed the presence absorption bands at 1665 and $1270 \mathrm{~cm}^{-1}$ attributed to $\mathrm{CON}$ and $\mathrm{C}=\mathrm{S}$, respectively.

The compound 2 and $o$-phenylenediamine dihydrochloride were successfully cyclized through the elimination of an ammonia molecule to the corresponding tetrazolo[5,1`:6,1]-1,2,4-triazino[4,5a] benzo[ $[d]$ imidazole (12). Both IR and ${ }^{1} \mathrm{H}$ NMR spectra showed no signals corresponding to the $\mathrm{NH}$ and $\mathrm{NH}_{2}$ groups thus, confirming the structure of compound $\mathbf{1 2}$ whereas its mass spectrum showed the moleculer ion peak at $m / z=210.86$ which was in agreement with molecular formula $\mathrm{C}_{9} \mathrm{H}_{5} \mathrm{~N}_{7}$ $(\mathrm{m} / \mathrm{z}=211)$.

Also, interaction of compound $\mathbf{2}$ with anthranilic acid under fusion conditions, the expected quinazolinone derivative $\mathbf{1 4}$ was resulted as the only isolable product. The formation of $\mathbf{1 4}$ was explained by the formation of unisolable intermediate 13, undergoes intramolecular ring closure to form the exactly product 14, which IR and ${ }^{1} \mathrm{H}$ NMR spectra exhibited devoid and $\mathrm{OH}$ and $\mathrm{NH}$ groups but showed CON absorption at $1670 \mathrm{~cm}^{-1}$ in the IR region. The mass spectrum of $\mathbf{1 4}$ showed a peak corresponding to its molecular ion at $m / z=239\left(\mathrm{C}_{10} \mathrm{H}_{5} \mathrm{~N}_{7} \mathrm{O}\right)$.

Moreover, treatment of two molar equivalents of cyclic imidoyl chloride $\mathbf{2}$ with one molor equivalent of each oxalic, malonic, and succinic acid dihydrazides caused a product in each case whose structure was verified from spectroscopic data. Its IR spectrum showed absorptions characteristic of $2 \mathrm{NH}$ and $\mathrm{CON}$ as well as two singlets attributed to $2 \mathrm{NH}$ each (exchangeable with $\mathrm{D}_{2} \mathrm{O}$ ) ${ }^{1} \mathrm{H}$ NMR signals. Accordingly, the previously products were decisively assigned as bishydrazides $15 a-c$. The aforementioned products were in accordance with previous report ${ }^{5}$ with cyclic imidoyl chlorides.

On the other hand the structures $\mathbf{1 5} a-c$ underwent cyclodehydration by phosphorus oxychloride to construct the bistriozolotetrazolotriazine derivatives $\mathbf{1 6} a-c$ which showed IR absorptions characteristic of only a $\mathrm{C}=\mathrm{N}$ and, most importantly, lacked any $\mathrm{NH}$ and $\mathrm{CON}$ absorption bands characteristic of the parent compounds $15 a-c$. Furthermore, the mass spectra of $\mathbf{1 6} a-c$ showed molecular ions in agreement with the assigned structures. Reaction ${ }^{17}$ of cyclic imidoyl chloride s with acid dihydrazides directly gave bistriazolo-structures.

\section{ANTIMicrobial SCREENing}

The newly prepared compounds were screened in vitro for their antimircrobial properties against Gram-positive (Staphylococcus aureus and Bacillus substilis) and Gram-negative (Klebsiella poneumoniae and Escherichia coli) bacteria strains and (Aspergillums niger and Candida albican) fungi strains. Ampicillin and Clotrimazole were used as standard drugs for bacteria and fungi, respectively. The minimal inhibitory concentration ${ }^{18}\left(M I C\right.$, in $\left.\mu \mathrm{g} . \mathrm{cm}^{-3}\right)$, and the results are summarized in Table 1 showing that 4, 6, 7a, 9a and 12 exhibit an antimicrobial activity against $S$. aureus (25\%); 3b, 6, 11, 12 and $16 \boldsymbol{a}$ against $B$. subtilis (25\%); 7a, 8, 14, 15a and 16c against $K$. poneumoniae $(50 \%) ; \mathbf{5}, \mathbf{6}, \mathbf{1 1}, \mathbf{1 2}$ and $\mathbf{1 6} \boldsymbol{a}(50 \%)$ while compound $\mathbf{3 b}$ possessed activity against $E$. coli comparable to that ampicillin. Moreover, 3a, 7c, 8 and 14 possessed an antimycotic activity against $A$. niger (50\%), and 4, 5, 11 and 12 against $C$. albican (50\%) comparable to that clotrimazole. The compound of $\mathbf{1 5} \boldsymbol{c}$ showed lower activity than the reference standards (ampicillin and clotrimazole) against the test organisms. 
Table1. Antimicrobial activity of synthesized compounds $\left(\mathrm{MIC} / \mu \mathrm{g} \mathrm{cm}^{-3}\right)$

\begin{tabular}{|l|c|c|c|c|c|c|}
\hline \multirow{2}{*}{ Compound } & \multicolumn{3}{|c|}{ Bacterial strain } & \multicolumn{2}{c|}{ Fungal strain } \\
\cline { 2 - 7 } & S.aureus & B.subtitis & K.pneumoniae & E.coli & A.niger & C.albican \\
\hline 3a & 100 & $>200$ & 100 & $>200$ & 25 & 100 \\
$\mathbf{4}$ & 100 & 50 & 100 & 25 & 100 & 100 \\
$\mathbf{5}$ & 50 & 100 & 100 & 100 & 100 & 25 \\
$\mathbf{6}$ & $>200$ & 100 & 100 & 50 & 100 & 25 \\
$\mathbf{7 a}$ & 50 & 50 & 100 & 50 & 100 & 100 \\
$\mathbf{7 c}$ & 50 & 100 & 50 & $>200$ & 100 & 100 \\
$\mathbf{8}$ & 100 & $>200$ & $>200$ & 100 & 25 & 100 \\
$\mathbf{9 a}$ & 100 & 100 & 50 & 100 & 25 & 100 \\
$\mathbf{1 1}$ & 50 & 100 & 100 & 100 & 100 & 100 \\
$\mathbf{1 2}$ & 100 & 50 & 100 & 50 & 100 & 25 \\
$\mathbf{1 4}$ & 50 & 50 & 100 & 50 & 100 & 25 \\
$\mathbf{1 5 a}$ & 100 & 100 & 50 & 100 & 25 & 100 \\
$\mathbf{1 5 c}$ & 100 & 100 & 50 & 100 & 100 & 100 \\
$\mathbf{1 6 a}$ & 100 & 100 & 100 & 100 & 100 & 100 \\
$\mathbf{1 6 c}$ & 100 & 50 & 100 & 50 & 100 & 100 \\
Ampicillin & 100 & 100 & 50 & $>200$ & 100 & 100 \\
Clotrimazole & 12.5 & 12.5 & 25 & 25 & -- & - \\
\hline
\end{tabular}

\section{EXPERIMENTAL}

\subsection{General}

Melting points were measured with a Gallenkamp apparatus and are uncorrected. The reactions were followed up and the purification of products was carried out on pre-(layer thickness $0.25 \mathrm{~mm}$; coated TLC plates Silica Gel-Merck), visualizin the spots in Iodine. IR spectra were recorded (KBr) on a Shimadzu FT-IR 8101 PC infrared spectrophotometer. The ${ }^{1} \mathrm{H}$ NMR spectra were determined in $\operatorname{DMSO}\left(d_{6}\right)$ at $300 \mathrm{MHz}$ on a Varian Mercury VX $300 \mathrm{NMR}$ spectrometer and their chemical shifts $(\delta / \mathrm{ppm})$ are reported using $T M S$ as internal standard. Mass spectra were recorded on a HP model MS 5988 spectrometer at electron ionizing energy of $70 \mathrm{ev}$. Elemtenal analyses were performed by the microanalytical Unit, Cairo University, Egypt.

\subsection{8-Chlorotetrazolo[5,1-f]-1,2,4-triazine (2)}

A suspension of tetrazolo[5,1-f]-1,2,4-triazin-8(7H)-one ${ }^{4}(\mathbf{1}, 0.006 \mathrm{~mol})$ and phosphorous pentachloride $(0.006 \mathrm{~mol})$ in phosphorous oxochloride $\left(10 \mathrm{~cm}^{3}\right)$ was heated under reflux on a waterbath for $2 \mathrm{~h}$. Then the mixture was cooled to room temperatue and poured into crushed ice-water slowly. The obtained solid was filtered off, washed with cold water, dried and recrystallized from abs. ethanol to give 2, yield 0.84g (74.34\%); m.p. 190-192 ${ }^{\circ} \mathrm{C} ;{ }^{1} \mathrm{H}$ NMR (DMSO- $d_{6}$ ) $\delta: 6.25 \mathrm{ppm}(\mathrm{s}, 1 \mathrm{H}$, $\mathrm{CH})$; MS: $m . z(\%)=156\left(\mathrm{M}^{+} \mathrm{Cl}^{35}, 11\right)$

Anal. Calcd. For $\mathrm{C}_{3} \mathrm{HClN}_{6}(156.5)$ : C, 23.00; H, 0.64; N, 53.67. Found: C, 22.61; H, 1.04; N, 53.34\%.

\subsection{General procedure for the synthesis of tetrazolotrazinyl amino acids $3 a, b$ and 4}

The corresponding amino acids $(0.006 \mathrm{~mol})$ namely: glycine, $\mathrm{D}$-alanine or $\beta$-alanine and sodium carbonate $(0.06 \mathrm{~mol})$ were dissolved in water $\left(20 \mathrm{~cm}^{3}\right)$. Then compound $2(0.006 \mathrm{~mol})$ was added to it and refluxed for $6 \mathrm{~h}$. The reaction mixture was left overnight at ambient temperature, and then treated with cold hydrochloric acid. The separated product was collected by filteration, dried and crystallized from abs. ethanol. The physico-chemical and spectra data of $\mathbf{3} a, b$ and $\mathbf{4}$ the following:

\subsection{2-(Tetrazolo[5,1-f]-1,2,4-triazin-8-ylamino)acetic acid (3a)}

Yield: 0.96g (77.05\%); m.p. 270-272 ${ }^{\circ} \mathrm{C}$; IR (KBr): v=3300 (OH), $2445(\mathrm{NH}), 1720 \mathrm{~cm}^{-1}(\mathrm{CO}) ;{ }^{1} \mathrm{H}$ NMR (DMSO- $\left.d_{6}\right): \delta=4.56\left(\mathrm{~s}, 2 \mathrm{H}, \mathrm{CH}_{2}\right), 6.26(\mathrm{~s}, 1 \mathrm{H}, \mathrm{CH}), 8.12\left(\mathrm{~s}, 1 \mathrm{H}, \mathrm{NH}\right.$, exchangeable with $\mathrm{D}_{2} \mathrm{O}$ ), $11.21 \mathrm{ppm}\left(\mathrm{s}, 1 \mathrm{H}, \mathrm{OH}\right.$ exchangeable with $\left.\mathrm{D}_{2} \mathrm{O}\right)$; MS: $\mathrm{m} / z(\%), 196\left(\mathrm{M}^{+}+1,16\right)$.

Anal. Calcd. for $\mathrm{C}_{5} \mathrm{H}_{5} \mathrm{~N}_{7} \mathrm{O}_{2}$ (195): C, 30.77; H, 2.56; N, 50.26. Found: C, 31.11; H, 2.41; N, 50.11\%.

\subsection{2-(Tetrazolo[5,1-f]-1,2,4-triazin-8-ylamino)propanoic acid (3b)}

Yield: 0.93g (69.66\%); m.p. 260-261 ${ }^{\circ} \mathrm{C}$; IR (KBr): v=3400(OH), $2430(\mathrm{NH}), 1705 \mathrm{~cm}^{-1}(\mathrm{CO}) ;{ }^{1} \mathrm{H}$ NMR (DMSO- $\left.d_{6}\right): \delta=1.51\left(\mathrm{~d}, 3 \mathrm{H}, \mathrm{CH}_{3}\right), 4.42(\mathrm{q}, 1 \mathrm{H}, \mathrm{CH}), 6.20(\mathrm{~s}, 1 \mathrm{H}, \mathrm{CH}), 8.22(\mathrm{~s}, 1 \mathrm{H}, \mathrm{NH}$, 
Synthesis, Structure, and Biological Screening of Some Condensed Heterocyclic Compounds from 8Chlorotetrazolo [5,1-f]-1,2,4-Triazine Precursor

exchangeable with $\left.\mathrm{D}_{2} \mathrm{O}\right), 10.83 \mathrm{ppm}\left(\mathrm{s}, 1 \mathrm{H}, \mathrm{OH}\right.$ exchangeable with $\left.\mathrm{D}_{2} \mathrm{O}\right) ; \mathrm{MS}: \mathrm{m} / z(\%)=211\left(\mathrm{M}^{+}+2\right.$, 20).

Anal. Calcd. For $\mathrm{C}_{6} \mathrm{H}_{7} \mathrm{~N}_{7} \mathrm{O}_{2}$ (209): C, 34.45; H, 3.35; N, 46.89. Found: C, 34.49; H, 3.31; N, 47.01\%.

\subsection{3-(Tetrazolo[5,1-f]-1,2,4-triazin-8-ylamino)propanoic acid (4)}

Yield: 0.88g (65.92\%); m.p. 268-270 ${ }^{\circ} \mathrm{C}$; IR (KBr): v=3300 (OH), $2395(\mathrm{NH}), 1720 \mathrm{~cm}^{-1}(\mathrm{CO}) ;{ }^{1} \mathrm{H}$ NMR (DMSO- $\left.d_{6}\right): \delta=3.22\left(\mathrm{t}, 2 \mathrm{H}, \mathrm{NCH}_{2}\right), 3.62\left(\mathrm{t}, 2 \mathrm{H}, \mathrm{CH}_{2} \mathrm{CO}\right), 6.25(\mathrm{~s}, 1 \mathrm{H}, \mathrm{CH}), 8.24(\mathrm{~s}, 1 \mathrm{H}, \mathrm{NH}$, exchangeable with $\left.\mathrm{D}_{2} \mathrm{O}\right), 11.02 \mathrm{ppm}\left(\mathrm{s}, 1 \mathrm{H}, \mathrm{OH}\right.$ exchangeable with $\left.\mathrm{D}_{2} \mathrm{O}\right)$; $\mathrm{MS}: m / z(\%)=209\left(\mathrm{M}^{+}\right.$, $6)$.

Anal. Calcd. For $\mathrm{C}_{6} \mathrm{H}_{7} \mathrm{~N}_{7} \mathrm{O}_{2}$ (209): C, 34.45; H, 3.35; N, 46.89. Found: C, 34.55; H, 3.48; N, 46.85\%.

4.7. General procedure for the synthesis of imidazotetrazolotriazines 5a,b and tetrazolo pyrimidotriazine 6

A mixture of the appropriate $(3 a, b$ or $4,0.005 \mathrm{~mol})$ and acetic anhydride $\left(20 \mathrm{~cm}^{3}\right)$, and anhydrous sodium acetate $(0.42 \mathrm{~g}, 0.005 \mathrm{~mol})$ was heated under reflux for $4 \mathrm{~h}$. The solvent was removed under reduced pressure, the obtained residue was washed with water the recrystallized from abs. ethanol. The following data of the title compounds were prepared according to this procedure are described:

4.8. Imidazo[1,2-d]tetrazolo[5,1-f]1,2,4-triazin-3(2H)-one (5a)

Yield: $0.58 \mathrm{~g}(63.74 \%)$; m.p. $250-251{ }^{\circ} \mathrm{C}$; IR (KBr): $v=1670 \mathrm{~cm}^{-1}(\mathrm{CON}) ;{ }^{1} \mathrm{H}$ NMR (DMSO- $\left.d_{6}\right): \delta=$ $4.66\left(\mathrm{~s}, 2 \mathrm{H}, \mathrm{CH}_{2}\right), 6.20 \mathrm{ppm}(\mathrm{s}, 1 \mathrm{H}, \mathrm{CH}), \mathrm{MS}: \mathrm{m} / z(\%)=179\left(\mathrm{M}^{+}+2,8\right)$.

Anal. Calcd. for $\mathrm{C}_{5} \mathrm{H}_{3} \mathrm{~N}_{7} \mathrm{O}$ (177): C, 33.90; H, 1.70; N, 55.37. Found: C, 33.81; H, 1.74; N, 55.41\%.

\subsection{2-Methylimidazo[1,2-d]tetrazolo[5,1-f]-1,2,4-triazin-3(2H)-one (5b)}

Yield: $0.62 \mathrm{~g}$ (68.13\%); m.p. $240-242{ }^{\circ} \mathrm{C}$; IR (KBr): $v=1675 \mathrm{~cm}^{-1}(\mathrm{CON}) ;{ }^{1} \mathrm{H}$ NMR (DMSO- $\left.d_{6}\right): \delta=$ $1.62\left(\mathrm{~d}, 3 \mathrm{H}, \mathrm{CH}_{3}\right), 4.60(\mathrm{q}, 1 \mathrm{H}, \mathrm{CH}), 6.22(\mathrm{~s}, 1 \mathrm{H}, \mathrm{CH}) \mathrm{MS}: \mathrm{m} / z(\%)=191\left(\mathrm{M}^{+}, 6\right)$.

Anal. Calcd. for $\mathrm{C}_{6} \mathrm{H}_{5} \mathrm{~N}_{7} \mathrm{O}$ (191): C, 37.70; H, 2.62; N, 51.31. Found: C, 38.01;

$\mathrm{H}, 2.44 ; \mathrm{N}, 51.52 \%$.

\subsection{Tetrazolo[5,1-f]pyrimido[1,2-d]-1,2,4-triazin-4(2H, 3H)-one (6)}

Yield: $0.52 \mathrm{~g}(57.14 \%)$; m.p. $245-246{ }^{\circ} \mathrm{C}$; IR (KBr): $v=1680 \mathrm{~cm}^{-1}(\mathrm{CON}) ;{ }^{1} \mathrm{H}$ NMR (DMSO- $\left.d_{6}\right): \delta=$ $3.10\left(\mathrm{t}, 2 \mathrm{H}, \mathrm{NCH}_{2}\right), 4.05\left(\mathrm{t}, 2 \mathrm{H}, \mathrm{COCH}_{2}\right), 6.28 \mathrm{ppm}(\mathrm{s}, 1 \mathrm{H}, \mathrm{CH}) \mathrm{MS}: \mathrm{m} / z(\%)=191\left(\mathrm{M}^{+}, 13\right)$.

Anal. Calcd. for $\mathrm{C}_{6} \mathrm{H}_{5} \mathrm{~N}_{7} \mathrm{O}$ (191): C, 37.70; H, 2.62; N, 51.31. Found: C, 37.42;

H, 3.01; N, $51.60 \%$.

\subsection{Reaction of 8-chlorotetrazolo[5,1-f]-1,2,4-triazine (2) with some acid hydrazides}

\subsubsection{General Procedure}

A mixture of compound $(\mathbf{2}, 0.006 \mathrm{~mol})$ and appropriate acid hydrazides $(0.006 \mathrm{~mol})$ namely: benzoic, $p$-toluic; $p$-chlorobenzoic, and $p$-nitrobenzoic in ethanol $\left(30 \mathrm{~cm}^{3}\right)$ was refluxed for $3 \mathrm{~h}$. after cooling the mass product was filtered off and recrystallized from abs. ethanol to provide $7 a-d$.

\subsection{3-Phenyl-1,2,4-triazolo[4,3-d]tetrazolo[5,1-f]-1,2,4-triazine (7a)}

Yield: $0.91 \mathrm{~g}$ (59.83\%); m.p. $210-211^{\circ} \mathrm{C}$; IR (KBr): $v=1620 \mathrm{~cm}^{-1}(\mathrm{C}=\mathrm{N}) ;{ }^{1} \mathrm{H}$ NMR (DMSO- $\left.d_{6}\right): \delta=$ 6.20 (s, 1H, CH), 7.02-8.52 ppm (m, 5H, ArH), MS: $m / z(\%)=240\left(\mathrm{M}^{+}+2,21\right)$.

Anal. Calcd. for $\mathrm{C}_{10} \mathrm{H}_{6} \mathrm{~N}_{8}$ (238): C, 50.42; H, 2.52; N, 47.06. Found: C, 50.55;

$\mathrm{H}, 2.31 ; \mathrm{N}, 46.82 \%$.

\subsection{3-p-Tolyl-1,2,4-triazolo[4,3-d]tetrazolo[5,1-f]-1,2,4-triazine $(7 b)$}

Yield: $0.84 \mathrm{~g}$ (52.17\%); m.p. $225-226{ }^{\circ} \mathrm{C}$; IR (KBr): $v=1625 \mathrm{~cm}^{-1}(\mathrm{C}=\mathrm{N}) ;{ }^{1} \mathrm{H}$ NMR (DMSO- $\left.d_{6}\right): \delta=$ $2.30\left(\mathrm{~s}, 3 \mathrm{H}, \mathrm{CH}_{3}\right), 6.25(\mathrm{~s}, 1 \mathrm{H}, \mathrm{CH}), 7.10-8.61 \mathrm{ppm}(\mathrm{m}, 4 \mathrm{H}, \mathrm{ArH}), \mathrm{MS}: m / z(\%)=252\left(\mathrm{M}^{+}, 17\right)$.

Anal. Calcd. for $\mathrm{C}_{11} \mathrm{H}_{8} \mathrm{~N}_{8}$ (252): C, 52.38; H, 3.18; N, 44.44. Found: C, 52.11; H, 3.22; N, 44.49\%.

\subsection{3-p-Chlorophenyl-1,2,4-triazolo[4,3- $d]$ tetrazolo[5,1-f]-1,2,4-triazine (7c)}

Yield: $0.94 \mathrm{~g}$ (54.02\%); m.p. $240-241^{\circ} \mathrm{C}$; IR (KBr): $v=1630 \mathrm{~cm}^{-1}(\mathrm{C}=\mathrm{N}) ;{ }^{1} \mathrm{H}$ NMR (DMSO- $\left.d_{6}\right): \delta=$ $6.20(\mathrm{~s}, 1 \mathrm{H}, \mathrm{CH}), 7.17-8.51 \mathrm{ppm}(\mathrm{m}, 4 \mathrm{H}, \mathrm{ArH}), \mathrm{MS}: m / z(\%)=237 \quad\left(\mathrm{M}^{+} \mathrm{Cl}^{35}, 8\right)$. 
Anal. Calcd. for $\mathrm{C}_{10} \mathrm{H}_{5} \mathrm{ClN}_{8}$ (272.5): $\mathrm{C}, 44.04 ; \mathrm{H}, 1.85 ; \mathrm{N}, 41.10$. Found: $\mathrm{C}, 44.10 ; \mathrm{H}, 1.81 ; \mathrm{N}$, $44.51 \%$.

\subsection{3-p-Nitrophenyl-1,2,4-triazolo[4,3- $d]$ tetrazolo[5,1-f]-1,2,4-triazine (7d)}

Yield: $1.11 \mathrm{~g}(61.33 \%)$; m.p. $270-272{ }^{\circ} \mathrm{C}$; IR (KBr): $v=1605 \mathrm{~cm}^{-1}(\mathrm{C}=\mathrm{N}) ;{ }^{1} \mathrm{H}$ NMR (DMSO- $\left.d_{6}\right): \delta=$ $6.27(\mathrm{~s}, 1 \mathrm{H}, \mathrm{CH}), 7.20-8.15 \mathrm{ppm}(\mathrm{m}, 4 \mathrm{H}, \mathrm{ArH}), \mathrm{MS}: m / z(\%)=284 \quad\left(\mathrm{M}^{+}+1,10\right)$.

Anal. Calcd. for $\mathrm{C}_{10} \mathrm{H}_{5} \mathrm{~N}_{9} \mathrm{O}_{2}$ (283): C, 42.40; H, 1.77; N, 44.52. Found: C, 42.61; H, 1.61; N, 44.20\%.

\subsection{3-Amino-1,2,4-triazolo[4,3-d]tetrazolo[5,1-f]-1,2,4-triazine (8)}

A mixture of compound $2(0.006 \mathrm{~mol})$ and thiosemicarbazide $(0.006 \mathrm{~mol})$ in abs. ethanol $\left(25 \mathrm{~cm}^{3}\right)$ was refluxed for $4 \mathrm{~h}$. The resulting product was cooled to ambient temperature and filtered, and recrystallized from abs. ethanol to yield 8 in $0.81 \mathrm{~g}$ (71.68\%); m.p. $220-221{ }^{\circ} \mathrm{C}$; $\mathrm{IR}(\mathrm{KBr}): v=3420$, $3390 \mathrm{~cm}^{-1}\left(\mathrm{NH}_{2}\right) ;{ }^{1} \mathrm{H}$ NMR (DMSO- $\left.d_{6}\right): \delta=6.20$

(s, $1 \mathrm{H}, \mathrm{CH}), 6.30 \mathrm{ppm}\left(\mathrm{s}, 2 \mathrm{H}, \mathrm{NH}_{2}\right.$, exchangeable with $\left.\mathrm{D}_{2} \mathrm{O}\right), \mathrm{MS}: \mathrm{m} / \mathrm{z}(\%)=177\left(\mathrm{M}^{+}, 22\right)$.

Anal. Calcd. for $\mathrm{C}_{4} \mathrm{H}_{3} \mathrm{~N}_{9}$ (177): C, 27.12; H, 1.70; N, 71.19. Found: C, 26.89; H, 1.95; N, 70.82\%.

\subsection{Preparation of 3-(N-arylideneamino)-1,2,4-triazolo[4,3-d]tetrazolo[5,1-f]-1,2,4-triazines $(9 \mathrm{a}-\mathrm{c})$}

Corresponding aromatic aldehyde $(0.006 \mathrm{~mol})$ namely: benzaldehyde,

$p$-tolualdehyde, and $p$-chlorobenzaldehyde was added to a solution of $8(0.006 \mathrm{~mol})$ in abs. ethanol $\left(20 \mathrm{~cm}^{3}\right)$. The reaction mixture was refluxed for $2 \mathrm{~h}$. The formed solid was collected by filtration, and recrystallized from abs. ethanol to result the title compounds $\mathbf{9} a-c$. The physico-chemical and spectra data as follows:

\subsection{3-(N-Benzylideneamino)-1,2,4-triazolo[4,3- $d]$ tetrazolo[5,1-f]-1,2,4-triazine $(9 a)$.}

Yield: $0.92 \mathrm{~g}$ (61.33\%); m.p. $190-192{ }^{\circ} \mathrm{C}$; IR (KBr): $v=1620 \mathrm{~cm}^{-1}(\mathrm{C}=\mathrm{N}) ;{ }^{1} \mathrm{H}$ NMR (DMSO- $\left.d_{6}\right): \delta=$ $6.22(\mathrm{~s}, 1 \mathrm{H}, \mathrm{CH}), 7.00-8.15 \mathrm{ppm}(\mathrm{m}, 5 \mathrm{H}, \mathrm{ArH}), 8.52 \mathrm{ppm}(\mathrm{s}, 1 \mathrm{H}, \mathrm{CH}=\mathrm{N}) \mathrm{MS}: \mathrm{m} / \mathrm{z}(\%)=265\left(\mathrm{M}^{+}, 30\right)$. Anal. Calcd. for $\mathrm{C}_{11} \mathrm{H}_{7} \mathrm{~N}_{9}$ (265): C, 49.81; H, 2.64; N, 47.55. Found: C, 50.11; H, 2.52; N, 46.40\%.

\subsection{3-(N-P-Tolylideneamino)-1,2,4-triazolo[4,3- $d]$ tetrazolo[5,1- $f]-1,2,4-$ triazine $(9 b)$}

Yield: $1.21 \mathrm{~g}$ (76.58\%); m.p. 185-187 ${ }^{\circ} \mathrm{C} ;{ }^{1} \mathrm{H}$ NMR (DMSO- $\left.d_{6}\right): \delta=2.33\left(\mathrm{~s}, 3 \mathrm{H}, \mathrm{CH}_{3}\right), 6.25(\mathrm{~s}, 1 \mathrm{H}$, $\mathrm{CH}), 7.20-8.15$ (m. 4H, ArH), $8.52 \mathrm{ppm}(\mathrm{s}, 1 \mathrm{H}, \mathrm{CH}=\mathrm{N}), \mathrm{MS}: \mathrm{m} / z(\%)=281\left(\mathrm{M}^{+}+2,9\right)$.

Anal. Calcd. for $\mathrm{C}_{12} \mathrm{H}_{9} \mathrm{~N}_{9}$ (279): C, 51.61; H, 3.23; N, 45.16. Found: C, 51.42; H, 3.72; N, 45.34\%.

\subsection{3-(N-P-Chlorobenzylideneamino)-1,2,4-triazolo[4,3- $d]$ tetrazolo[5,1-f]-1,2,4-triazine $(9 c)$}

Yield: $1.11 \mathrm{~g}$ (65.68\%); m.p. $197-199{ }^{\circ} \mathrm{C}$; IR (KBr): $v=1628 \mathrm{~cm}^{-1}(\mathrm{C}=\mathrm{N}) ;{ }^{1} \mathrm{H}$ NMR (DMSO- $\left.d_{6}\right): \delta=$ 6.21 (s, 1H, CH), 7.27-8.20 (m. 4H, ArH), 9.02 ppm (s, 1H, CH=N).

Anal. Calcd. for $\mathrm{C}_{11} \mathrm{H}_{6} \mathrm{ClN}_{9}$ (299.5): C, 44.07; H, 2.00; N, 42.07. Found: C, 43.88; H, 2.34; N, $42.13 \%$.

\subsection{3-Phenyl-2-thioxo-2,3-dihydrotetrazolo[5 $[1$ :6,1]-1,2,4-triazino[4,5-a]-1,3,5-triazin-4-one} (11)

The solution of ammonium thiocyanate $(0.006 \mathrm{~mol})$ in abs. ethanol was added to a stirred solution of $2(0.006 \mathrm{~mol})$ in abs. ethanol. The reaction mixture was stirred for $30 \mathrm{~min}$ at ambient temperature. Ammonium chloride was precipitated during the reaction, filteration and phenyl isocyanate $(0.006$ mol) was added to the filtrate ad the reaction mixture was heated under reflux for 30 min after cooling the precipitated was collected and crystallized from abs. ethanol, and dried, yield: $1.32 \mathrm{~g}$ (69.47\%); m.p. $235-237^{\circ} \mathrm{C} ; \quad \operatorname{IR}(\mathrm{KBr}): v=1665(\mathrm{CON}), 1270 \mathrm{~cm}^{-1}(\mathrm{C}=\mathrm{S}) ;{ }^{1} \mathrm{H}$ NMR $\left(\mathrm{DMSO}-d_{6}\right): \delta=$ 6.25 (s, 1H, CH), 7.30-8.35 ppm (m, 5H, ArH); MS: $m / z(\%)=300\left(\mathrm{M}^{+}+2,19\right)$.

Anal. Calcd. for $\mathrm{C}_{11} \mathrm{H}_{6} \mathrm{~N}_{8} \mathrm{OS}$ (298): $\mathrm{C}, 44.30 ; \mathrm{H}, 2.01 ; \mathrm{N}, 37.58$. Found: $\mathrm{C}, 44.72 ; \mathrm{H}, 1.99 ; \mathrm{N}$, $37.99 \%$.

\subsection{Tetrazolo[5 $, 1: 6,1]-1,2,4$-triazino $[4,5-a]$ benzo $[d]$ imidazole (12)}

A mixture of compound $2(0.006 \mathrm{~mol})$ and $o$-phenylenediamine dihydrochloride $(0.006 \mathrm{~mol})$ was fused in an oil bath at $190-191{ }^{\circ} \mathrm{C}$ for an hour and then allowed to attain ambient temperature and added to $20 \mathrm{~cm}^{3}$ of cold water. The mass product obtained was filtered off, dried, and recrystallized 
Synthesis, Structure, and Biological Screening of Some Condensed Heterocyclic Compounds from 8Chlorotetrazolo [5,1-f]-1,2,4-Triazine Precursor

from abs. ethanol to produce 12. yield: $0.82 \mathrm{~g}(65.08 \%)$; m.p. $210-212{ }^{\circ} \mathrm{C} ;{ }^{1} \mathrm{H}$ NMR (DMSO- $\left.d_{6}\right): \delta=$ 6.24 (s, 1H, CH), 7.20-8.12 ppm (m, 4H, ArH); MS: m/z (\%) = $210.86\left(\mathrm{M}^{+}, 26\right)$.

Anal. Calcd. for $\mathrm{C}_{9} \mathrm{H}_{5} \mathrm{~N}_{7}$ (211): C, 51.18; H, 2.37; N, 46.45. Found: C, 50.98; H, 2.11; N, 46.31\%.

\subsection{Tetrazolo[5 , $\left.1^{\prime}: 6,1\right]-1,2,4$-triazino[4,5-b]quinazolin-8-one (14)}

A mixture of $2(0.006 \mathrm{~mol})$ and anthranilic acid $(0.006 \mathrm{~mol})$ was heated in oil bath at $190-191{ }^{\circ} \mathrm{C}$ for an hour and then allowed to cool down at room temperature; the obtained residue was crystallized from abs. ethanol to result 14. yield: $0.94 \mathrm{~g}(61.44 \%)$; m.p. $220-222{ }^{\circ} \mathrm{C}$; $\mathrm{IR}(\mathrm{KBr}): v=1670(\mathrm{CON}),{ }^{1} \mathrm{H}$ NMR (DMSO- $\left.d_{6}\right): \delta=6.27$ (s, $\left.1 \mathrm{H}, \mathrm{CH}\right), 7.05-8.10 \mathrm{ppm}(\mathrm{m}, 4 \mathrm{H}, \mathrm{ArH}) ; \mathrm{MS}: \mathrm{m} / z(\%)=239\left(\mathrm{M}^{+}, 22\right)$.

Anal. Calcd. for $\mathrm{C}_{10} \mathrm{H}_{5} \mathrm{~N}_{7} \mathrm{O}$ (239): C, 50.21; H, 2.09; N, 41.00. Found: C, 49.91; H, 2.11; N, 41.33\%.

\subsection{Synthesis of 15a-c: General Procedure}

To a solution of $2(0.006 \mathrm{~mol})$ in $15 \mathrm{~cm}^{3}$ abs. ethanol, a solution of $(0.006 \mathrm{~mol})$ corresponding acid dihydrazide namely: oxalic, malonic and succinic in $15 \mathrm{~cm}^{3}$ abs, ethanol was gradually added, and the mixture was heated under reflux at $100{ }^{\circ} \mathrm{C}$ for an hour. The product which separated upon cooling was filtered off, and crystallized from mixture of water and abs. ethanol to afford the products $15 a-c$.

\subsection{Oxalyl bis\{(tetrazolo[5,1-f]-1,2,4-triazin-8-yl) hydrazide $\}(15 a)$}

Yield: $1.67 \mathrm{~g}$ (72.93\%); m.p. $230-232{ }^{\circ} \mathrm{C}$; IR (KBr): $v=3285,3280(2 \mathrm{NH}), 1665 \mathrm{~cm}^{-1}(\mathrm{CON}),{ }^{1} \mathrm{H}$ NMR $\left(\right.$ DMSO- $\left.d_{6}\right): \delta=6.15,6.20$, (2s, $1 \mathrm{H}$ each, 2CH); 11.90, $12.20 \mathrm{ppm}(2 \mathrm{~s}, 2 \mathrm{H}$ each, $2 \mathrm{NH}$ each, exchangeable with $\left.\mathrm{D}_{2} \mathrm{O}\right)$; $\mathrm{MS}: m / z(\%)=360\left(\mathrm{M}^{+}+2,27\right)$.

Anal. Calcd. for $\mathrm{C}_{8} \mathrm{H}_{6} \mathrm{~N}_{16} \mathrm{O}_{2}$ (358): C, 26.82; H, 1.68; N, 62.57. Found: C, 27.25; H, 1.71; N, 62.67\%.

\subsection{Malonyl bis $\{$ (tetrazolo[5,1-f]-1,2,4-triazin-8-yl) hydrazide $\}(15 b)$}

Yield: $1.45 \mathrm{~g}$ (60.92\%); m.p. 220-222 ${ }^{\circ} \mathrm{C} ;{ }^{1} \mathrm{H}$ NMR (DMSO- $d_{6}$ ): $\delta=4.41$ (s, $2 \mathrm{H}, \mathrm{CH}_{2}$ ); 6.20 (s, $2 \mathrm{H}$, $2 \mathrm{CH}), 11.85,12.30 \mathrm{ppm}\left(2 \mathrm{~s}, 2 \mathrm{H}\right.$ each, $2 \mathrm{NH}$ each, exchangeable with $\left.\mathrm{D}_{2} \mathrm{O}\right) ; \mathrm{MS}: \mathrm{m} / z(\%)=372\left(\mathrm{M}^{+}\right.$, $10)$.

Anal. Calcd. for $\mathrm{C}_{9} \mathrm{H}_{8} \mathrm{~N}_{16} \mathrm{O}_{2}$ (372): C, 29.03; H, 2.15; N, 60.22. Found: C, 28.86; H, 2.22; N, 60.52\%.

\subsection{Succinyl bis\{(tetrazolo[5,1-f]-1,2,4-triazin-8-yl) hydrazide $\}(15 c)$}

Yield: $1.34 \mathrm{~g}$ (54.25\%); m.p. $240-243{ }^{\circ} \mathrm{C}$; IR (KBr): $v=3275,3290(2 \mathrm{NH}), 1690 \mathrm{~cm}^{-1}(\mathrm{CON}),{ }^{1} \mathrm{H}$ NMR $\left(\right.$ DMSO- $\left.d_{6}\right): \delta=3.25,3.40$ (2t, $2 \mathrm{H}$ each, $\mathrm{CH}_{2}$ each); 6.25 (s, 2H, 2CH), 12.01, $12.20 \mathrm{ppm}(2 \mathrm{~s}, 2 \mathrm{H}$ each, $2 \mathrm{NH}$ each, exchangeable with $\left.\mathrm{D}_{2} \mathrm{O}\right)$; $\mathrm{MS}: m / z(\%)=387\left(\mathrm{M}^{+}+1,9\right)$.

Anal. Calcd. for $\mathrm{C}_{10} \mathrm{H}_{10} \mathrm{~N}_{16} \mathrm{O}_{2}$ (386): C, 31.09; H, 2.59; N, 58.03. Found: C, 30.82; H, 2.61; N, $57.82 \%$.

\subsection{Synthesis of 16a-c: General Procedure}

The respective of $\mathbf{1 5 a - c}(0.006 \mathrm{~mol})$ was treated with $20 \mathrm{~cm}^{3}$ phosphorus oxychloride and heated under reflux for $2 \mathrm{~h}$. After attaining ambient temperature, the mixture was poured onto a cold saturated solution of sodium bicarbonate and the crude solid which separated was filtered off, washed with water, dried, and crystallized from abs. ethanol to yield the products $\mathbf{1 6} a-c$.

\subsection{Bis $\{1,2,4-$ triazolo[4,3-d]tetrazolo $[5,1-f]-1,2,4-$ triazin-3-yl $\}(16 a)$}

Yield: 1.62g (78.64\%); m.p. $240-242{ }^{\circ} \mathrm{C}$; IR (KBr): $v=1610 \mathrm{~cm}^{-1}(\mathrm{C}=\mathrm{N}), \mathrm{MS}: \mathrm{m} / z(\%)=323\left(\mathrm{M}^{+}+1\right.$, 10).

Anal. Calcd. for $\mathrm{C}_{8} \mathrm{H}_{2} \mathrm{~N}_{16}$ (322): C, 29.81; H, 0.62; N, 69.57. Found: C, 29.90; H, 1.05; N, 70.01\%.

\subsection{Bis $\{1,2,4-$ triazolo[4,3-d] tetrazolo $[5,1-f]-1,2,4-$ triazin-3-yl $\}$ methane $(16 b)$}

Yield: $1.72 \mathrm{~g}(80.00 \%) ;$ m.p. $230-232{ }^{\circ} \mathrm{C} ;{ }^{1} \mathrm{H}$ NMR (DMSO- $\left.d_{6}\right): \delta=4.46,\left(\mathrm{~s}, 2 \mathrm{H}, \mathrm{CH}_{2}\right), 6.25 \mathrm{ppm}(\mathrm{s}$, $2 \mathrm{H}$ each, 2CH); MS: $m / z(\%)=336\left(\mathrm{M}^{+}, 27\right)$.

Anal. Calcd. for $\mathrm{C}_{9} \mathrm{H}_{4} \mathrm{~N}_{16}$ (336): C, 32.14; H, 1.19; N, 66.67. Found: C, 32.09; H, 1.14; N, 67.09\%. 


\subsection{Bis $\{1,2,4-$ triazolo $[4,3-d]$ tetrazolo $[5,1-f]-1,2,4-$ triazin-3-yl $\}$ ethane $(16 c)$}

Yield: $1.76 \mathrm{~g}$ (78.57\%); m.p. $250-271{ }^{\circ} \mathrm{C}$; IR (KBr): $v=1620 \mathrm{~cm}^{-1}(\mathrm{C}=\mathrm{N})$, MS:

$m / z(\%)=351\left(\mathrm{M}^{+}+1,19\right)$.

Anal. Calcd. for $\mathrm{C}_{10} \mathrm{H}_{6} \mathrm{~N}_{16}$ (350): C, 34.29; H, 1.71; N, 64.00. Found: C, 34.09;

H, $1.95 ; \mathrm{N}, 64.24 \%$.

\section{ANTIMICROBIAL SCREENING}

Solutions of the test compounds, ampicillin trihydrate and clotrimazole were prepared in DMSO at a concentration of $100 \mu \mathrm{g} / \mathrm{cm}^{3}$. Twofold dilution of the compounds were prepared $(800,400, \ldots 6.25$ $\left.\mathrm{g} / \mathrm{cm}^{3}\right)$. The microorganism suspensions at $10^{6}$ colony Formin Unit $1 \mathrm{~cm}^{3}\left(C F U / \mathrm{cm}^{3}\right)$ concentration were inoculate to the corresponding wells. Plates were incubated at $36^{\circ} \mathrm{C}$ for 24 to $28 \mathrm{~h}$ the incubation chamber. The minimal inhibitory concentration $(M I C)$ were determined. Controls with DMSO and infected media were also investigated.

\section{CONCLUSION}

The foregoing results demonstrated the utility of 8-chlorotetrazolo [5,1-f]-1,2,4-triazine as synthons for the construction of some condensed heterocyclic nitrogen structures by different cyclization reagents. The antibacterial and antifungi activities of the synthesized compounds were even comparable to ampicillin and clotrimazole.

\section{REFERENCES}

[1] (a) Gein, V. L.; Zamaraeva, T. M.; Dmitriev, M. V., Russ. J. Org. Chem., 2016, 52558. (b) Golobokova, T. V.; Vereshchagin, L. I., Ratovskii, G. V.; Proidakov, A. G.; Kizhnyaev, V. N. Ibid. 2016, 52, 1039.

[2] El Badry, S. M.; Taha, M. A. M. J. Korean Chem. Soc., 2014, 58, 381.

[3] El-Badry, S.M.; Taha, M.A.M. J. Korean. Chem. Soc. 2011, 55, 974.

[4] Taha, M.A.M.; El-Badry, S.M. J. Korean. Chem. Soc. 2010 , 54, 414.

[5] Taha, M.A.M.; El-Badry, S.M. Monatsh. Chem. 2008, 139, 1261.

[6] Taha, M.A.M. Phosphorus, Sulphur, Silicon Relat. Elem. 2008, 183, 2525.

[7] Taha, M.A.M. Monatsh. Chem. 2007, 138, 505.

[8] Taha, M.A.M.; El-Badry, S.M. Phosphorus, Sulfur, Silicon Relat Elem 2007, 182, 1011.

[9] Taha, M.A.M.; El-Badry, S.M. J. Chin. Chem. Soc. 2006, 53, 1181.

[10] Taha, M.A.M. J. Chin. Chem. Soc. 2005, 52, 137.

[11] Moderhack, D. J. Prakt. Chem.1998, 340, 687.

[12] Kolodobskii, G. I.; Ostrovskii, V. A.; Popavskii, V. S. Chem. Heterocycl. Cpds. 1981, 17, 965.

[13] Karnik, A.V.; Malviya, N.J.; Kulkarni, A.M.; Jadhav, B.L. Eur. J. Med. Chem. 2006, 41, 891.

[14] Jantova, S.; Ruzekova, I.; Stantovsky, S.; Spirkova, K. Neoplasma 1997, 44, 240.

[15] Rubat, C.; Coudert, P.; Couquelet, J.M.; Tronche, P.; Bastide, J.; Bastide, P. Formaco 1990, 45, 331.

[16] Afify, A. A.; El-Nady, S.; Sayed, M. A.; Mohey, I. Indian J. Chem. 1988, $27 B, 920$.

[17] Shaban, M. A. E.; Taha, M.A. M.; Nazr, A. Z., J. Islamic Acad. Sci, 1990, 3, 98.

[18] Murray, P.R.; Baron, E.J.; Pfaller, M.A.; Tenover, F.C.; Yolken R.T. Manual of Clinical Microbiology. In: Antimicrobial Agents and Susceptibility Testing. Woods, G. L., Washington J.A., Eds.; ASM Press: Wahington DC, 1995, 1327. 